American Journal of Applied Sciences 7 (3): 408-414, 2010

ISSN 1546-9239

(C) 2010Science Publications

\title{
Application of Trujillo Algorithm for Analyzing of Structures
}

\author{
H. Hashamdar and Z. Ibrahim \\ Department of Civil Engineering, University of Malaya, Kuala Lumpur, Malaysia
}

\begin{abstract}
Problem statement: The Trujillo algorithm is indirect method and has been used in this study. The approach is based upon the principle of conservation of energy. Approach: Cable structures are very light and flexible and they undergo appreciable deflections when subjected to external loading. Results: Since all the main load-carrying members in cable structures are usually in tension, there are no stability problems and the strength of high tensile steel used for cables can be rationally utilized. Conclusion/Recommendations: In order to define the position of equilibrium the method of analysis should, therefore, cater for the change in geometry caused by any form of applied load. Hence the classical linear theories of structural mechanisms cannot be used for the solution of cable assemblies.
\end{abstract}

Key words: Nonlinear dynamic response optimization, structural, optimization, nonlinear dynamic analysis

\section{INTRODUCTION}

There are a number of methods for nonlinear static analysis of cable structures, such as Tottenham (Chahande and Arora, 1994). Those carry out theoretical analysis using the continuous membrane approach. Tension structure may also be analyzed as discrete system then we can pull a result, the number of equations obtained has an unknown's finite number.

In the discrete systems, tension structures are regarded as assemblies of finite number members connected at joints or nodes. And also nonlinear equations are set up for the condition of joint equilibrium in term of the joint displacements from which the equilibrium displacements can be found using an iterative process.

Although a number of method have been developed for dynamic response analysis of structural system, but there are only a few methods which can be used for nonlinear dynamic response analysis.

Tension roof structures are first analyzed for static loads and then their dynamic response is checked to ensure that the design provides sufficient safety and structural serviceability. Although cable structures, under service loads, exhibit nonlinear behavior, recent developments on the computing methods have made it possible to carry out the analysis with great accuracy.

Basic methods to determine the equilibrium position of cables for static loads are now well known. Cable systems may be treated either as (a) continuous or (b) discrete idealizations.
They are described in (Buchholdt, 1985; Park, 2007; Kang et al., 2006) and are the following: (a) the method of steepest descent, (b) the method of conjugate gradient (Fletcher and Reeves, 1964; Fletcher and Powell, 2003), (c) the method of Newton-Raphson (Clough and Wilson, 2003), (d) the method of RungeKutta and (e) the method of Fletcher-Powell. Methods (a-c) are most commonly used. This study has shown that the Trujillo algorithm for minimization total energy in dynamic work is suitable for analyzing nonlinear structures with a large number of joints.

Equation of motion for a system: The equation of motion for a multi degree (MDOF) system can be written as:

$$
M \ddot{X}+C(t) \dot{X}+k(T) X=P(T)
$$

Where:

$\mathrm{M}=$ Mass matrix

$\mathrm{C}(\mathrm{t})=$ Damping matrix

$\mathrm{K}(\mathrm{t})=$ Stiffness matrix

$\mathrm{X}=$ Displacement vector

$\dot{\mathrm{X}}=$ Velocity vector

$\ddot{\mathrm{X}}=$ Acceleration vector

$P(t)=$ Load vector

For a Single Degree Of Freedom (SDOF) system the equation of motion becomes: 


$$
\mathrm{m} \ddot{\mathrm{X}}+\mathrm{c}(\mathrm{t}) \dot{\mathrm{X}}+\mathrm{k}(\mathrm{t}) \mathrm{x}=\mathrm{P}(\mathrm{t})
$$

Where:

$\mathrm{m}=$ Mass of the system

$\mathrm{c}(\mathrm{t})=$ Time varying damping

$\mathrm{k}(\mathrm{t})=$ Time varying stiffness

$\mathrm{x}=$ Displacement

$\dot{\mathrm{X}}=$ Velocity

$\ddot{\mathrm{X}}=$ Acceleration

$p(t)=$ time varying Load

The assumption of a constant mass in the case of both SDOF and MDOF system is arbitrary as it could be represented as a time varying quantity.

Since $m$ is a non-zero constant value, both sides of Eq. 2 can be divided by $m$ and for:

$$
\begin{aligned}
& P=\frac{C(t)}{m} \\
& Q=\frac{K(t)}{m} \\
& F=\frac{p(t)}{m}
\end{aligned}
$$

Equation 2 can be written as:

$$
\ddot{\mathrm{X}}+\mathrm{P} \dot{\mathrm{X}}+\mathrm{QX}=\mathrm{F}
$$

This is a second order different equation.

The mathematical solution of Eq. 3 depends on the values of $\mathrm{P}, \mathrm{Q}$ and $\mathrm{F}$. Equation 3 is a linear differential equation if $\mathrm{P}$ and $\mathrm{Q}$ are independent of $\mathrm{x}$ and remains so even if $\mathrm{P}$ and $\mathrm{Q}$ are functions of $\mathrm{t}$.

In such cases there are well known methods for solving Eq. 3.

The solution normally given in the form $x=f(t)$ and gives exact values of $x$ for any t. once $f(t)$ is defined $x$ and $x$ can be derived by differentiation.

When $P$ and $Q$ are functions of $x$ and $f$ is either a function of $t$ or given as a table of values Eq. 3 becomes non-linear.

For such equation the solution cannot be expressed in functional form and it is necessary to plot or tabulate to solution curve point by point, beginning at $\left(\mathrm{t}_{0}, \mathrm{x}_{0}\right)$ and then at selected intervals of $t$, usually equally spaced, until the solution has been extended to cover the required range.
Note: In this study it is assumed that F is independent of $x$.

Thus the solutions of non-linear equations require a step-by-step approach and are normally based on the use of interpolation or finite difference equations.

The independent variable $t$ is divided into equal intervals $\Delta t$, over the range of the desired solution. Thus the variables after $\mathrm{n}$ and $(\mathrm{n}+1)$ intervals are given by $t_{n}=n . \Delta t$ and $t_{n+1}=(n+1) \Delta t$ respectively.

All the quantities so far defined, such as $\mathrm{C}(\mathrm{t}), \mathrm{c}(\mathrm{t})$, $K(t), k(t), X$, $x$, will be designed to a time $t_{n}$ by the subscript n, e.g., $C_{n}, c_{n}, K_{n}, k_{n}, X_{n}, X_{n}$ and at times $t_{n+1}$ by the subscript $\mathrm{n}+1$.

At time $t_{n}$ it is assumed that the values of all the parameters are known as well as the values for same parameters at all previous intervals $(\mathrm{n}-1),(\mathrm{n}-2), \ldots \ldots, 2,1$.

At time $t_{n+1}$ it is assumed that the values of the variable parameters are not known and that the purpose of the analysis is to find the value of $x_{n+1}$ (in the case of MDOF system, $X_{n+1}$ ) and its derivatives which satisfy:

$$
\mathrm{mX}_{\mathrm{n}+1}+\mathrm{c}_{\mathrm{n}+1} \mathrm{X}_{\mathrm{n}+1}+\mathrm{k}_{\mathrm{n}+1} \mathrm{X}_{\mathrm{n}+1}=\mathrm{p}_{\mathrm{n}+1}
$$

At time $t_{n}$ and time $t_{n+1}=t_{n}+\Delta t$ the condition of dynamic equilibrium requires respectively that:

$m \ddot{X}_{n}+C X_{n}+k_{n} X_{n}=p_{n}$

and

$$
m \ddot{X}_{n+1}+C_{n+1} \dot{X}_{n+1} 1+k_{n+1} X_{n+1}=p_{n+1}
$$

Since:

$$
\begin{aligned}
\mathrm{X}_{\mathrm{n}+1} & =\mathrm{X}_{\mathrm{n}}+\Delta \mathrm{X} \\
\dot{\mathrm{X}}_{\mathrm{n}+1} & =\dot{\mathrm{X}}_{\mathrm{n}}+\Delta \dot{\mathrm{X}} \\
\ddot{\mathrm{X}}_{\mathrm{n}+1} & =\ddot{\mathrm{X}}_{\mathrm{n}}+\Delta \ddot{\mathrm{X}} \\
\mathrm{C}_{\mathrm{n}+1} & =\mathrm{C}_{\mathrm{n}}+\Delta \mathrm{C} \\
\mathrm{K}_{\mathrm{n}+1} & =\mathrm{K}_{\mathrm{n}}+\Delta \mathrm{K}
\end{aligned}
$$

Equation 6 may be written as:

$$
\mathrm{m} \Delta \ddot{\mathrm{X}}_{\mathrm{n}}+\mathrm{c}_{\mathrm{n}} \Delta \dot{\mathrm{X}}_{\mathrm{n}}+\mathrm{k}_{\mathrm{n}} \Delta \mathrm{X}_{\mathrm{n}}+\mathrm{R}_{2}=\mathrm{R}_{1}
$$

Basic assumption and step-by step integration of equation of motion: Evaluation these expression at the end of the time interval when $t=\Delta t$.

Leads to the following expressions for the incremental velocity and displacement: 
$\Delta \dot{\mathrm{X}}=\ddot{\mathrm{X}}_{\mathrm{n}} \Delta \mathrm{t}+\frac{1}{2} \Delta \ddot{\mathrm{X}} \Delta \mathrm{t}$

$\Delta \mathrm{X}=\dot{\mathrm{X}}_{\mathrm{n}} \Delta \mathrm{t} \frac{1}{2} \Delta \ddot{\mathrm{X}}_{\mathrm{n}} \Delta \mathrm{t}^{2}+\frac{1}{6} \Delta \ddot{\mathrm{X}} \Delta \mathrm{t}^{2}$

In general it has been found to be convenient to use the incremental displacement as the basic variable and hence the $\Delta \mathrm{x}$ and $\Delta \mathrm{x}$ in terms of $\Delta \mathrm{x}$. rearranging Eq. $9 \mathrm{a}$ and $\mathrm{b}$ yields:

$$
\begin{aligned}
& \Delta \ddot{\mathrm{X}}=\frac{6}{\Delta \mathrm{t}} \Delta \mathrm{X}-\frac{6}{\Delta \mathrm{t}} \Delta \dot{\mathrm{X}}_{\mathrm{n}}+3 \dot{\mathrm{X}}_{\mathrm{n}} \\
& \Delta \dot{\mathrm{X}}=\frac{3}{\Delta \mathrm{t}} \Delta \mathrm{X}-3 \dot{\mathrm{X}}_{\mathrm{n}}-\frac{\Delta}{2} \ddot{\mathrm{X}}_{\mathrm{n}}
\end{aligned}
$$

Substitution of Eq. 10 into Eq. 8 and assuming k and $\mathrm{c}$ remain constant during the time interval leads to:

$$
\begin{aligned}
& \left(\mathrm{m} \frac{6}{\Delta \mathrm{t}} \Delta \mathrm{X}+\frac{3}{\Delta \mathrm{t}} \Delta \mathrm{X}+\mathrm{k}_{\mathrm{n}}\right) \Delta \mathrm{X} \\
& =\mathrm{m}\left(\frac{6}{\Delta \mathrm{t}} \Delta \dot{\mathrm{X}}_{\mathrm{n}}+3 \dot{\mathrm{X}}\right)+\mathrm{C}_{\mathrm{n}}\left(3 \dot{\mathrm{X}}_{\mathrm{n}}+\frac{\Delta \mathrm{t}}{2} \ddot{\mathrm{X}}\right)+\mathrm{R}_{1}
\end{aligned}
$$

This equation can be solved for $\Delta \mathrm{t} \cdot$ and then $\Delta \mathrm{t}$ can be calculated using Eq. 10.

The Newmark method-beta: The Newmark-beta method (Kang et al., 2006) is a method of numerical integration used to solve differential equations. It is used in finite element analysis to model dynamic systems.

Newmark (1998) has expressed the velocities and displacements at the end of a time increment in terms of the known parameters at the beginning and the unknown acceleration at the end of the time step as:

$$
\begin{aligned}
& \dot{X}_{n+1}=\dot{X}_{n}+(1-\gamma) \ddot{X}_{n} \Delta t+\gamma \ddot{X}_{n+1} \Delta t \\
& X_{n+1}=X_{n}+\dot{X}_{n} \Delta t+\left(\frac{1}{2}-\beta\right) \ddot{X}_{n} \Delta t^{2}+\beta \ddot{X}_{n+1} \Delta t^{2}
\end{aligned}
$$

where, $\gamma$ and $\beta$ are parameters which can be varied at will.

The value of $\gamma$ is taken to be equal to $\frac{1}{2}$ as other values will produce numerical damping. Eq. 13 can therefore be written as:

$$
\dot{\mathrm{X}}_{\mathrm{n}+1}=\dot{\mathrm{X}}_{\mathrm{n}}+\frac{1}{2} \ddot{\mathrm{X}}_{\mathrm{n}} \mathrm{n} \Delta \mathrm{t}+\frac{1}{2} \ddot{\mathrm{X}}_{\mathrm{n}+1} \Delta \mathrm{t}
$$

In addition to the expressions for the displacement and velocities the condition of dynamic equilibrium at the end of the time interval:

$M \ddot{X}_{n+1}+C_{n+1} \dot{X}_{n+1}+K_{n+1} X_{n+1}=P_{n+1}$

Yield the following expression for the acceleration at the end of the time step:

$$
\ddot{X}_{n+1}=M^{-1}\left[P_{n+1}-C_{n+1} X_{n+1}-\dot{K}_{n+1} X_{n+1}\right]
$$

Equation 14-16 from the basis for the non-linear analysis of structural systems using the Newmark method.

\section{MATERIALS AND METHODS}

Trujillo's method: Trujillo presented an explicit algorithm for the dynamic response analysis of structural system in 1977 (Trujillo, 1977) and has tested the method for linear problems. For linear undamped systems the method was shown to be unconditionally stable.

An algorithm based upon Trujillo's method has been developed for non-linear systems by Surya Kumar and Knudson (1979) but does not take into account the effect of damping.

The Trujillo algorithm: Trujillo splits the stiffness and damping matrices into upper and lower triangular forms, as indicated below, by the subscripts $\mathrm{U}$ and $\mathrm{L}$ respectively and presents, without giving the development of the equations, the following algorithm which is divided into a forward and a backward substitution.

Forward substitution:

$$
\begin{aligned}
& X_{n+1 / 2}=\left[M+\frac{\Delta t}{2} C_{L}+\frac{\Delta t^{2}}{8} K_{L}\right]^{-1} . \\
& \left(\begin{array}{l}
{\left[M+\frac{\Delta t}{2} C_{L}-\frac{\Delta t^{2}}{8} K_{U}\right] X_{n}} \\
+\left[M+\frac{\Delta t}{4}\left(C_{L}-C_{U}\right)\right] \frac{\Delta t}{2} \dot{X}_{n}+\frac{\Delta t^{2}}{16}\left[P_{n+1}+P_{n}\right]
\end{array}\right)
\end{aligned}
$$




$$
\dot{X}_{n+1 / 2}=\left[X_{n+1 / 2}-X_{n}\right] \frac{4}{\Delta t}-\dot{X}_{n}
$$

Backward substitution:

$$
\begin{aligned}
& X_{n+1}=\left[M+\frac{\Delta t}{2} C_{U}+\frac{\Delta t^{2}}{8} K_{U}\right]^{-1} \\
& \left(\begin{array}{l}
{\left[M+\frac{\Delta t}{2} C_{U}-\frac{\Delta t^{2}}{8} K_{L}\right] X_{n+1 / 2}+} \\
\left.\left[M+\frac{\Delta t}{4}\left(C_{U}-C_{L}\right)\right] \frac{\Delta t}{2} \dot{X}_{n+1 / 2}+\frac{\Delta t^{2}}{16}\left[P_{n+1}+P_{n}\right]\right) \\
\dot{X}_{n+1}=\left[X_{n+1}-X_{n+1 / 2}\right] \frac{4}{\Delta t}-\dot{X}_{n+1 / 2}
\end{array}\right)
\end{aligned}
$$

An advantage of this algorithm is that, since it is restricted to the use of diagonal mass matrices only, the coefficient matrices of $X_{n+1 / 2}$ and $X_{n+1}$ are obtained respectively in upper and lower triangular forms. Thus the solution of the equations at time $t_{n+1 / 2}$ is reduced to forward and at time $t_{n+1}$ to backward substitution only.

Trujillo suggests two ways of splitting the stiffness and damping matrices.

The first is a symmetric splitting which satisfies the conditions:

$$
\mathrm{K}_{\mathrm{L}}+\mathrm{K}_{\mathrm{U}}=\mathrm{K} ; \mathrm{K}_{\mathrm{L}}=\mathrm{K}_{\mathrm{U}}^{\mathrm{T}}
$$

And

$$
\mathrm{C}_{1}+\mathrm{C}_{\mathrm{u}}=\mathrm{C} ; \mathrm{C}_{\mathrm{L}}=\mathrm{C}_{\mathrm{U}}^{\mathrm{T}}
$$

The second way differs from the first only by the manner in which the diagonal elements are distributed.

Kumar, who extended Trujillo's work to apply to non-linear systems, but excludes damping and thus reduces the equilibrium equations at the $\mathrm{n}^{\text {th }}$ step to:

$$
M \ddot{X}_{n}+R_{n}=P_{n}
$$

where, $R_{n}$ is the internal force vector and presents the following algorithm for the middle and the end of step:

$$
\begin{aligned}
& \dot{X}_{n+1 / 2}=\left[M+\frac{\Delta t^{2}}{8} K_{L}\right]^{-1} \cdot\left(\begin{array}{l}
\left.M-\frac{\Delta t^{2}}{8} K_{L}\right] \dot{X}_{n} \\
-\frac{\Delta t}{2} R_{n}+\frac{\Delta t}{2} P_{n}
\end{array}\right) \\
& X_{n+1 / 2}=X_{n}+\frac{\Delta t}{4}\left[\dot{X}_{n}-\ddot{X}_{n+1 / 2}\right] \\
& \dot{X}_{n+1 / 2}=\left[M+\frac{\Delta t^{2}}{4} K_{U}\right]^{-1} \cdot \\
& \quad\left(\left[M-\frac{\Delta t^{2}}{8} K_{U}\right] \dot{X}_{n+1 / 2}-\frac{\Delta t}{2} R_{n+1 / 2}+\frac{\Delta t}{2} P_{n+1 / 2}\right) \\
& X_{n+1}=X_{n+1 / 2}+\frac{\Delta t}{4}\left[\dot{X}_{n+1 / 2}+\dot{X}_{n+1}\right]
\end{aligned}
$$

Non-linearity is taken in to account by updating the stiffness matrix at the end and if necessary also at the middle of each time step.

For non-linear problems the algorithm loses its unconditional stability and becomes similar to the Newmark $(\beta=1 / 4)$ method. The size of the time step is, however, mainly governed by the required accuracy.

\section{RESULTS AND DISCUSSION}

Numerical experimentation: The analytical method is used to experiment with mathematical models. The mathematical chosen was a $7 * 5$ flat net with Endcaster of freedom (Fig. 1 and 2).

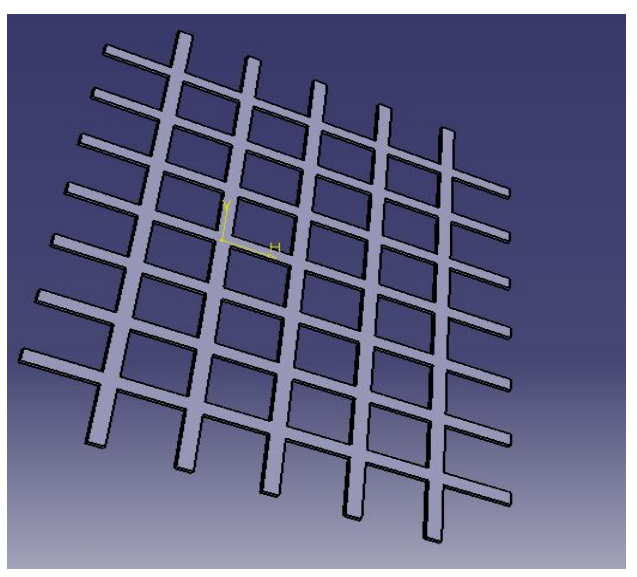

Fig. 1: 7*5 flat net 


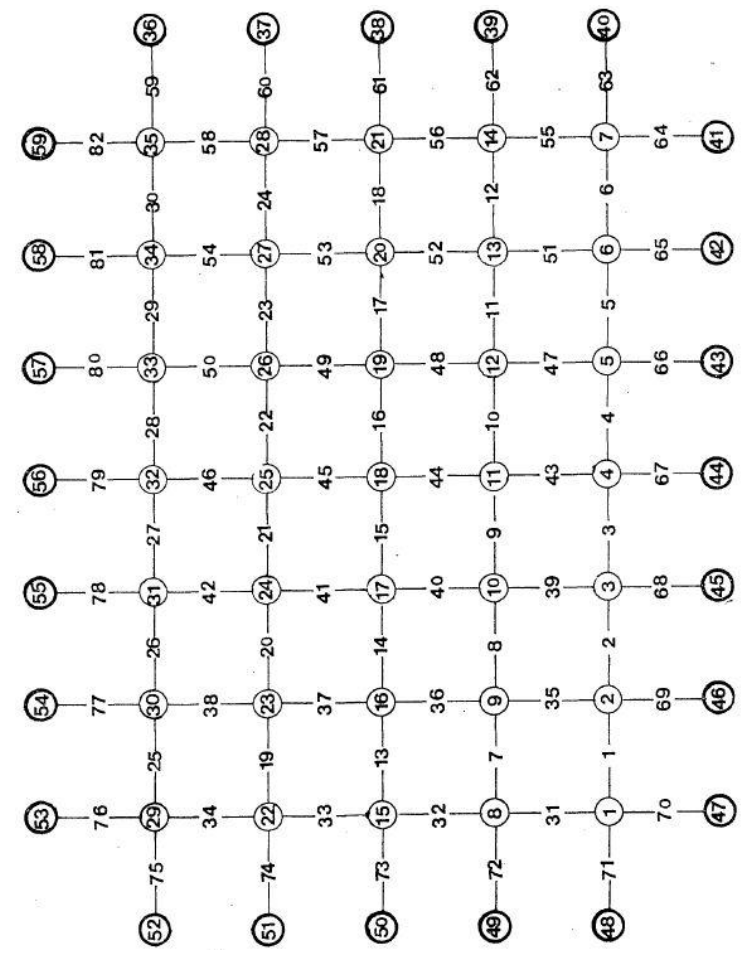

Fig. 2: $7 * 5$ flat net

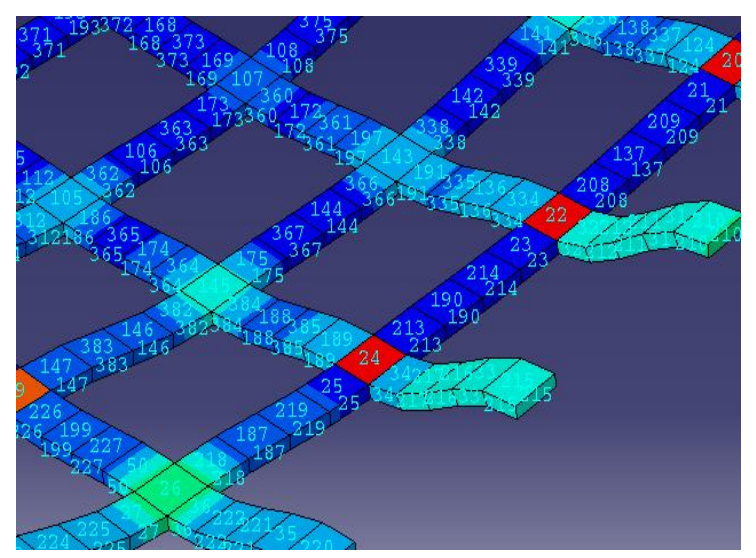

Fig. 3: Mesh: Hex of element shape

The $7 * 5$ net was also built as an experimental model and tested in order to verify the static and dynamic nonlinear theories given in this study (Fig. 1 and 2).

The analytical method is used to experiment with mathematical models in Fig. 1-3 and also Newton analytical, Effect of load, and computing time ratio is shown in Fig. 4-6. The 7*5 net was also built as an experimental model and tested in order to verify the static and dynamic nonlinear theories given in this study.

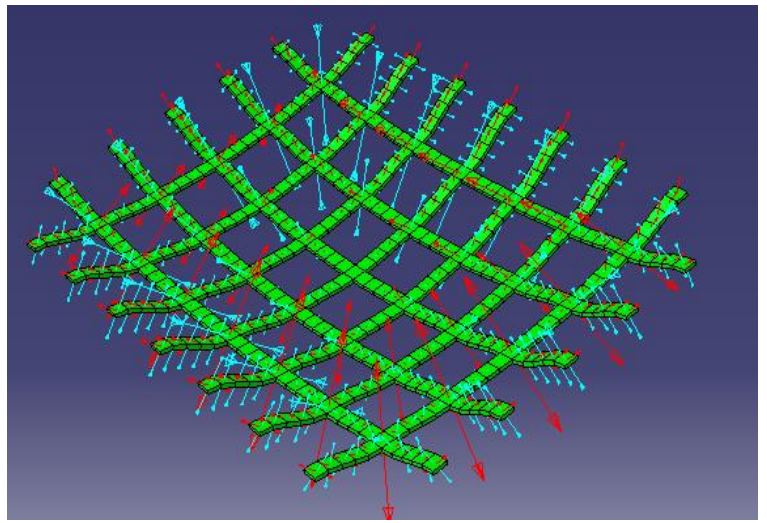

Fig. 4: Effect of load on flat net

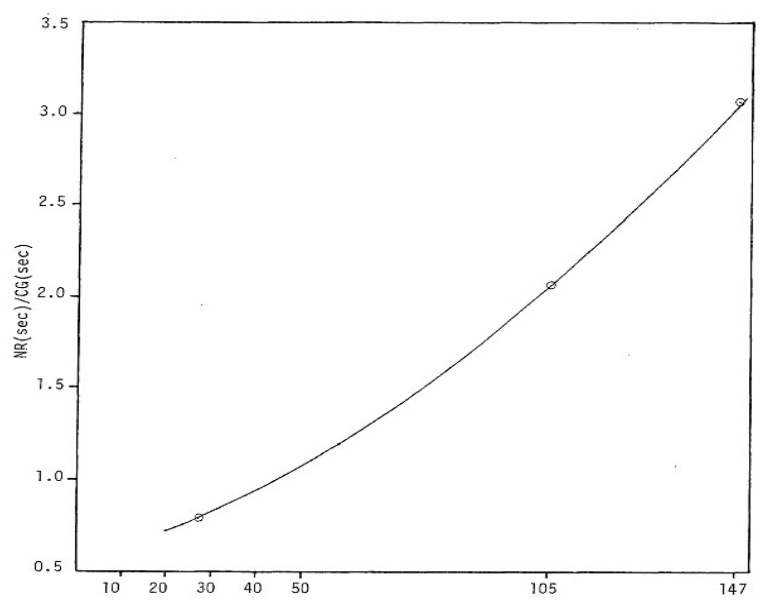

Fig. 5: Variation of NR/TA computing time. Ratio with degrees of freedom for a $5 \mathrm{sec}$; Response calculation; (NR) Newton-Raphson; (TA) Trujillo Algorithm

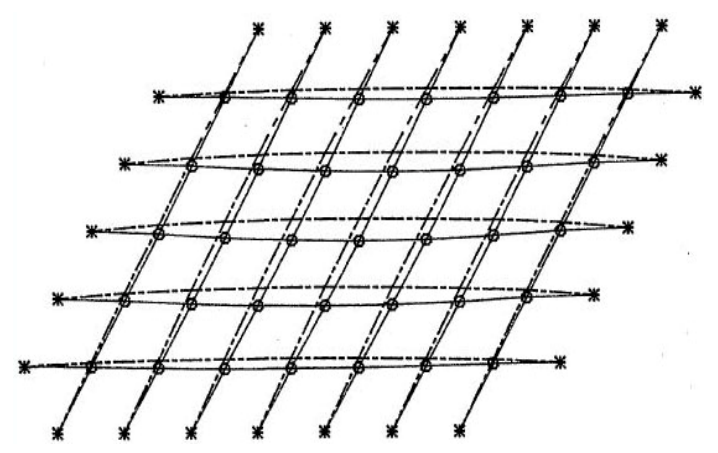

Fig. 6: $7 * 5$ flat net, mode1

In this case, explicit is specified and also 3D stress on family of mesh is selected. Geometric linear and quadratic is recognized. 


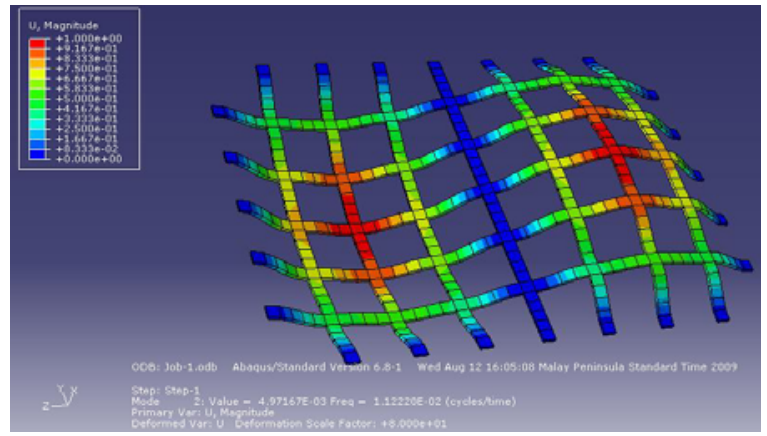

Fig. $7 \mathrm{a}: 7 * 5$ flat net, mode 2

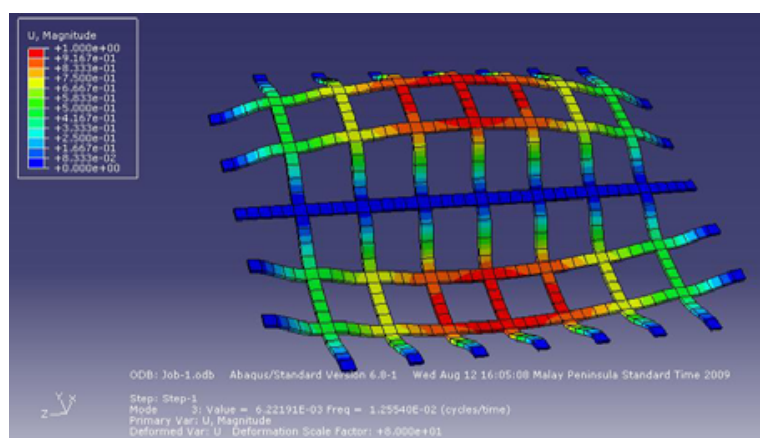

Fig. $7 b: 7 * 5$ flat net, mode 3

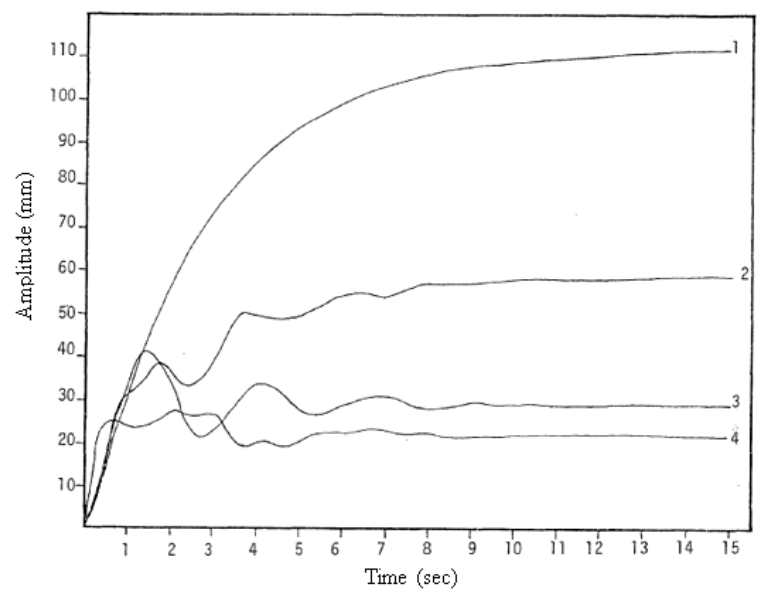

Fig. 8: $7 * 5$ flat, mode 3

The percentage differences between the theoretical and numerical testing results did not in any case exceed $10 \%$, this is thought to be acceptable.

The damping matrix for the proposed theory is calculated separately and its calculation does not affect the formulation of the theory in present form.
It was possible to use an orthogonal damping matrix in which the damping ratio could be varied in the different mode (Fig. 7a, b and 8).

A mode shape describes the expected curvature (or displacement) of a surface vibrating at a particular mode.

Increases of degree of freedom in Trujillo algorithm are very slowly and then changes of degree of freedom are reasonable.

Build up of the amplitude of joint 6 from $t=0$ to steady state vibration:

- Newton-Raphson method (linear)

- Newton-Raphson method (nonlinear)

- Trujillo algorithm method (linear)

- Trujillo algorithm method (nonlinear)

\section{CONCLUSION}

The object of this study was principally to develop a linear dynamic analysis theory for the analysis of tension structure subjected to dynamic loading and verify the theory by Numerical experimentation

The propose method was found to be stable for time steps equal to less or less than half the smallest periodic time of the system. The numerical experimentation carry out by static and dynamic testing of flat net showed a good agreement between the numerical experimentation results and the theoretically predicted values.

The behavior of each method has been extensively investigated and compared with each other. Buchholdt et al. (1974) in his initial work on cable structures used both the direct and relaxed steepest descent methods and found them to be inefficient in term of computational time. Ryu et al. (1985) and other have used the Newton-Raphson method with and without modifications, to solve the resulting set of nonlinear equations for cable beams and nets.

The Newton-Raphson method founds that method converged rapidly near the solution, but that a slow start made it rather costly to use because of the matrix inversion or complete solution of equations required separate iteration and the method when applied to function with a larger number of variables requires considerable computer storage to store the Hessian.

In this study has shown the stability of Trujillo algorithm method to reduce the number of iterations. The nonlinearity of the energy function makes the use of the Trujillo algorithm and Trujillo algorithm converge more rapidly to the neighborhood of the solution such as Fletcher algorithms. 


\section{REFERENCES}

Buchholdt, H.A., 1985. An Introduction to Cable Roof Structures. Cambridge University Press, Cambridge, pp: 257.

Buchholdt, H.A., N.K. Das and A.J. Al-Hilli, 1974. A gradient method for the analysis of cable structures with flexible boundaries. Proceeding of the International Conference on Tension Roof Structure, (ICTRS'74), Polytechnic of Central London.

Chahande, A.I. and J.S. Arora, 1994. Optimization of large structure subjected to dynamic loads with the multiplier method. Int. J. Numer. Methods Eng., 37: 413-430.

Clough, R.W. and E.L. Wilson, 2003. Dynamic analysis of large structural systems with local nonlinearities. Comput. Meth. Applied Mech. Eng., 17: 107-123.

Fletcher, R. and C.M. Reeves, 1964. Function minimization by conjugate gradients. Comput. J., 7: 149-153.

Fletcher, R. and M.J.D. Powell, 2003. Large deflection analysis of prestressed networks. ASCE J. Struct. Div., 98: 633-653.
Kang, B.S., G.J. Park, J.S. Arora, 2006. A review of optimization of structures subjected to dynamic loads. Struct. Multidis. Optimiz., 31: 81-95.

Newmark, N.M., 1998. A method of computations for structural dynamic. J. Eng. Mech. Div., ASCE, 85: 67-94.

Park, G.J., 2007. Analytical Methods for Design Practice. Springer, Germany, ISBN: 1461-3562, pp: 627.

Ryu, Y.S., M. Haririan, C.C. Wu, J.S. Arora, 1985. Structural design sensitivity analysis of nonlinear response. Comput. Struct., 21: 245-255.

Surya Kumar, G.V. and W.C. Knudson, 1979. Application of Trujilo's algorithm for nonlinear dynamics. Proceeding of the International Conference on Computer Applications in Civil Engineering, (ICCACE'79), University of Roorkee, India, pp: 55.

Trujillo, D.M., 1977. An unconditionally stable explicit algorithm for structural dynamics. Int. J. Numer. Methods Eng., 11: 1579-1592. 